\title{
A parallel finite-volume spatial/angular agglomeration multigrid method for radiative heat transfer computation
}

\author{
G. N. Lygidakis \& I. K. Nikolos \\ School of Production Engineering and Management, \\ Technical University of Crete, Greece
}

\begin{abstract}
The development of a spatial/angular agglomeration multigrid methodology is reported for the acceleration of a parallel node-centered finite-volume algorithm, numerically predicting radiative heat transfer in tetrahedral or hybrid unstructured grids. For spatial agglomeration, a sequence of coarser meshes is constructed, by merging the adjacent control volumes on a topology-preserving framework. Similarly, for the angular agglomeration, coarser angular resolutions are generated with the fusion of the neighbouring solid control angles, deriving a new angular discretization with the quarter number of control angles. The multigrid accelerated numerical solution of the Radiative Transfer Equation (RTE) is achieved via the Full Approximation Scheme (FAS) in a V-Cycle process. The proposed algorithm has been validated against benchmark test cases, demonstrating its capability for improved computational performance, especially in problems with purely scattering media and/or reflecting surfaces.

Keywords: radiative heat transfer, node-centered, finite-volume method, multigrid, spatial/angular agglomeration, 3D unstructured hybrid grids.
\end{abstract}

\section{Introduction}

Radiation is one of the major modes of heat transfer in many engineering applications including combustion, such as combustion chambers, buildings on fire, etc. For its numerical prediction various methodologies have been developed during the last decades with the finite-volume method, initially introduced by Raithby and Chui [1], being one of the most widely applied techniques. Part of its popularity derives from its capability to be implemented 
along with unstructured grids, employed for the representation of complicated geometries. Nevertheless, comparing to the structured solvers for the same test case, the unstructured ones seem to be relatively inefficient [2-4]. Moreover, using a finer spatial and angular discretization, higher-order accurate schemes $[5,6]$ or a higher-order RTE [7] reduces even more the computational performance of such codes.

A remedy to this drawback appears to be a well established technique in Computational Fluid Dynamics (CFD), the multigrid method, which considers the solution of the examined problem on successively coarser meshes [4]. Its development was based on the observation that iterative schemes converge more slowly on finer grids, as they succeed efficient damping for the high frequency errors but not for the low frequency ones [8]; using coarser meshes the latter become actually high frequency ones, allowing for their quick damping by the commonly implemented numerical approaches $[4,8]$. Various types of the multigrid methodology have been developed during the past decades, such as the Algebraic Multigrid (AMG), considering the construction of a coarsening matrix rather than the generation of any new grid topology, the geometrical multigrid, according to which new independent or nested meshes are generated, and the agglomeration multigrid introduced initially by Lallemand [9] and analyzed in this paper. For a multigrid method involving grid generation, the procedure allowing for the interaction between each two successively coarser grids has to be defined. For example, the Full Multigrid (FMG) method begins with the solution of the governing equations on the coarsest grid, in order to use it as an initial guess for the solution on the next finer one [4, 8], while the FAS, used in the present work, considers the relaxation of the finest mesh solution on all the available grids during each cycle, utilizing appropriate transfer operators for their interaction; in that way smoothed versions of the finest level solution are obtained from the coarser grids [8]. Independently of the employed scheme, an associating relation is required for smoothing the variables from the finer to the coarser mesh (restriction) as well as for interpolating them from the coarser to the finer one (prolongation) [8].

In this study, a spatial, an angular, and a combined spatial/angular agglomeration multigrid method was developed to be used along with an existing parallel node-centered finite-volume algorithm for the numerical computation of radiative heat transfer through absorbing-emitting and scattering gray media $[5,10]$. For the spatial scheme, an isotropic agglomeration of the neighbouring control volumes is considered on a topology-preserving framework resembling the advancing front technique [11]. The procedure is performed simultaneously on all the partitions in which the computational field has been divided for parallelization [10], beginning from the boundary nodes and extending to the internal region. Special care is attributed to the nodes constituting the overlapping area, allowing for interaction between the sub-domains. The numerical solution is achieved implementing the FAS via a V-cycle strategy $\mathrm{V}(1,0)$, which corresponds to relaxation only once before the restriction to the coarser level, and to none after the prolongation to the finer one [11]. Similarly, for the angular method, the adjacent solid control angles are merged, deriving a 
coarser directional "sphere" $[5,10]$ with the quarter number of control angles; the same technique with the spatial scheme is implemented for numerical computation. Finally, a combined spatial/angular multigrid method was developed for even more acceleration, which involves the nested implementation of the pre-mentioned angular scheme at each step of the spatial one. The proposed methodology, which to the authors' best knowledge hasn't been employed before, was validated against benchmark test cases, revealing its potential for improved computational performance, especially in problems with purely scattering media and/or reflecting surfaces accounting for increased interaction between the defined solid control angles.

\section{Radiative heat transfer solver}

The radiative intensity $I_{p}(\vec{r}, \hat{s})$ at a node $p$ in position $\vec{r}$ and time $t$ along a path $\hat{s}$ through an absorbing, emitting and scattering gray medium can be defined by the time-dependent RTE as [5]

$$
\frac{1}{c} \frac{d I_{p}(\vec{r}, \hat{s})}{d t}+\frac{d I_{p}(\vec{r}, \hat{s})}{d s}=-\left(k_{\alpha}+\sigma_{s}\right) I_{p}(\vec{r}, \hat{s})+S_{R}^{\vec{r}, \hat{s}} .
$$

where $c$ is the propagation speed of radiation in the medium, $k_{a}$ is the absorption coefficient, $\sigma_{s}$ is the scattering coefficient and $S_{R}^{\vec{r}, \hat{s}}$ is the sum of the emissive black body source term and the in-scattering integral $[5,10]$. According to the basic idea of the finite-volume method, the RTE is solved for a finite number of control volumes and a finite number of solid control angles, calling for spatial and angular discretization of the examined computational domain respectively. Thus, a node-centered median dual control volume strategy is employed, considering the construction of the control volume of a node by connecting lines defined by edge midpoints, barycenters of faces and barycenters of elements, sharing this node $[4,5,10]$. For the angular discretization, the commonly used "sphere" to represent the directional domain is divided into a finite number of solid control angles, using lines of constant longitude and constant latitude $[1,5]$.

The integration of eqn (1) over the control volume $V_{p}$ of a node $p$ and the discrete control angle $\Delta \Omega^{m n}$, along with the implementation of the divergence theorem on the left hand side terms, derives the following formulation [5]

$$
\Delta I_{p}^{m n} \frac{V_{p} \Delta \Omega^{m n}}{c \Delta t}+\sum_{i} I_{i}^{m n} D_{c i}^{m n} \Delta A_{i}=\left[-\left(k_{\alpha}+\sigma_{s}\right) I_{p}^{m n}+S_{R}^{m n}\right] V_{p} \Delta \Omega^{m n}
$$

where $D_{c i}^{m n}$ is the directional weight of the $i$ part of the control volume's surface (extending to an area $\Delta A_{i}$ ) along the path of the solid control angle $m n$. For the computation of the source term $S_{R}^{m n}$ in case of an anisotropically scattering medium, the Legendre polynomials [10] are employed to define the average 
scattering phase function at a pre-computation stage, as their value does not differentiate during the iterative procedure. The intensity value at the interface of each two adjacent control volumes is associated with the corresponding nodal values by implementing the step scheme as follows [5]

$$
I_{i}^{m n} D_{c i}^{m n}=I_{p}^{m n} D_{c i, o u t}^{m n}+I_{q}^{m n} D_{c i, i n}^{m n} .
$$

where the first right hand side directional weight corresponds to an outgoing intensity of the control volume of node $p$, while the second one to an incoming intensity from the control volume of node $q$. Considering the edge-based structure of the algorithm, the pre-mentioned flux computation becomes a straightforward procedure, performed with a single loop over all the edges of the grid. For the overhang problem, resulting from the implementation of the finitevolume method on an unstructured mesh, the pixelation method is applied [5].

Finally, the contribution of the boundary conditions have to be included to the boundary nodes' flux balance; for opaque and diffusive surfaces, as these employed in this work, the radiative intensity $I_{w}^{m n}$ coming into the boundary control volume is defined as [5]

$$
I_{w}^{m n}=\varepsilon_{w} I_{b, p}+\frac{1-\varepsilon_{w}}{\pi} \sum_{m_{i}=1}^{N_{\theta}} \sum_{n_{i}=1}^{N_{\varphi}} I_{p}^{m_{i} n_{i}} D_{c i, o u t, w}^{m_{i} n_{i}} .
$$

where $\varepsilon_{w}$ is the wall emissivity, $N_{\theta}$ is the number of azimuthal angles and $N_{\varphi}$ the number of polar ones. The first term accounts for the black body intensity of the wall node while the second one for the diffusively returning intensity from the same surface. Considering an implicit treatment of the boundary conditions [5] along with the pre-mentioned step scheme, eqn (2) for a node $p$ is reformulated as

$$
\begin{aligned}
& \Delta I_{p}^{m n} \frac{V_{p} \Delta \Omega^{m n}}{c \Delta t}=\left[-\left(k_{\alpha}+\sigma_{s}\right) I_{p}^{m n}+S_{R}^{m n}\right] V_{p} \Delta \Omega^{m n}, \\
& -\sum_{i} I_{p}^{m n}\left(D_{c i, \text { out }}^{m n} \Delta A_{i}+D_{c i, \text { out }, w}^{m n} \Delta A_{w}\right)-\sum_{i}\left(I_{q}^{m n} D_{c i, \text { in }}^{m n} \Delta A_{i}+I_{w}^{m n} D_{c i, i n, w}^{m n} \Delta A_{w}\right)=R_{p}^{m n}
\end{aligned}
$$

where $R_{p}^{m n}$ is the flux balance of node $p$ along the path $m n$. Depending on the examined test case a second-order spatial scheme with an appropriate slope limiter [5] can be used, replacing the values of radiative intensity $I_{p}^{m n}$ and $I_{q}^{m n}$ at the right hand side of the previous equation with their corresponding Taylor series' reconstructed values $[4,5]$. Finally, for time integration and relaxation of eqn (5), a second-order accurate in time, four-stage Runge-Kutta method (RK) is used, along with the local time-stepping technique, allowing for the utilization of the maximum allowable time step for each node $[4,5]$. 


\section{Spatial agglomeration multigrid scheme}

For the implementation of the spatial agglomeration multigrid scheme, first of all the fusion strategy for the generation of the coarser grids' sequence has to be defined. As previously mentioned, the agglomeration is performed on a topology-preserving framework at each partition, following pre-defined rules, concerning mainly the fusion of the boundary nodes as well as the ghost nodes at the overlapping regions [10]. The whole procedure can be divided in the following steps:

a) The nodes belonging to two or more boundary-condition-type closures (excluding those with symmetry conditions) are identified and transferred to the next agglomeration level as singletons.

b) A list of the eligible for fusion wall boundary nodes, the so-called seed nodes, is constructed.

c) The main agglomeration procedure begins by looping over these nodes and merge their control volumes with those of their adjacent ones, belonging to the same surface (all at the same boundary only, or all being internal ones), creating the supernodes of the next coarser level, unless they have been already merged or limited for agglomeration. In case a singleton-supernode is created, due to the previous fusion of all its neighbours or in case a supernode is completely surrounded by another supernode, it is examined to be merged with its adjacent supernode with the less number of included nodes. The procedure stops when all the seed nodes have been agglomerated or assigned as singletons.

d) A new list of seed nodes is created, including the nodes that their control volumes have been contacted by the agglomeration front. In addition, a priority hierarchy is imposed, based on the number of times a node has been touched by the pre-mentioned front, as well as on the minimum number of adjacent nodes.

e) The steps $c$ and $d$ are repeated until all the nodes of the computational grid have been examined for agglomeration. In Figure 1 the fusion of three control volumes at a prismatic region is illustrated.

f) The ghost nodes at the overlapping area are agglomerated or assigned as singletons, according to their core nodes' fusion at the adjacent partition, creating ghost supernodes which may include different number of merged nodes compared to their corresponding core supernodes.

g) The new superedges, connecting adjacent supernodes, are created. The internal edges at each supernode's control volume are deleted, while the external ones are maintained for the computation of the new directional weights.

h) Finally, the supernodes assigned as singletons are identified, while their neighbours are marked to become also singletons at the next agglomeration level; the generation of significantly irregular coarser grids is avoided.

i) If an even coarser mesh is required, the whole procedure is repeated. 


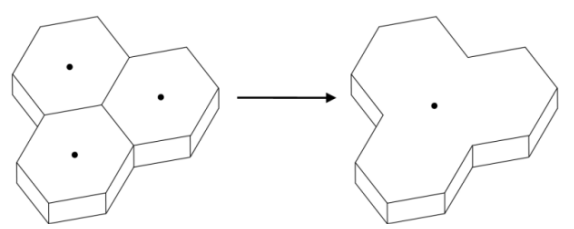

Figure 1: Spatial agglomeration of three control volumes at a prismatic region.

Since the generation of coarser grids is completed, the corresponding directional weights, pixelation method coefficients, and local time steps are computed, in order the FAS scheme to be employed and the multigrid accelerated solution of the RTE to be obtained. In accordance with this scheme, at each multigrid cycle eqn (5) is relaxed for the first non-multigrid level (denoted at next with the subscript $h$ ), while at next the values of radiative intensity as well as the flux balances are restricted to the next coarser level (denoted with subscript $H$ ), using the transfer operators $\left(I_{I}\right)_{h}^{H}$ and $\left(I_{R}\right)_{h}^{H}$ as [4]

$$
\begin{aligned}
& I_{P, \text { restricted }}^{m n}=\left(I_{I}\right)_{h}^{H} I_{p}^{m n}=\sum I_{p}^{m n} \cdot V_{p} / V_{P} . \\
& R_{P, \text { restricted }}^{m n}=\left(I_{R}\right)_{h}^{H} R_{p}^{m n}=\sum R_{p}^{m n} .
\end{aligned}
$$

where $P$ corresponds to the supernode including nodes $p$ of the finer grid. As mentioned earlier in Introduction, an approximated first-order accurate spatial RTE is solved for the coarser grids, in which the forcing function $A_{H}$ is added to the right hand side term of eqn (5), reformulating it as follows [4]:

$$
R_{P, F A S}^{m n}=R_{P}^{m n}\left(I_{P}^{m n}\right)+\underbrace{\left[R_{P, \text { restricted }}^{m n}-R_{P}^{m n}\left(I_{P, \text { restricted }}^{m n}\right)\right]}_{A_{H}} .
$$

In case an even coarser level $2 H$ has been generated, eqns (6)-(8) are again employed, until an updated solution on the coarsest grid is obtained. Subsequently, the corrections of radiative intensity are prolongated to the next finer level, using a simple point injection scheme as [4]:

$$
\Delta I_{p}^{m n}=\left(I_{I}\right)_{H}^{h} \Delta I_{P}^{m n}=\Delta I_{P}^{m n}=I_{P}^{m n}-I_{P, \text { restricted }}^{m n} .
$$

The new value of radiative intensity at the next finer grid is computed by adding this correction, while the procedure continues until the corresponding values at the finest resolution are updated.

\section{Angular agglomeration multigrid scheme}

The angular scheme begins with the fusion of the neighbouring solid control angles similarly to the spatial one; nevertheless, it is much simpler due to the absence of the limitations constraining the latter one. At each angular agglomeration level, every two adjacent azimuthal and polar angles are identified and merged respectively, deriving a coarser directional "sphere" with the quarter 
number of solid control angles (half number of control angles in two dimensions). In Figure 2 the schematic representation of the angular fusion in two dimensions is illustrated; the 16 control angles of the initial resolution are merged to 8 ones for the second level.

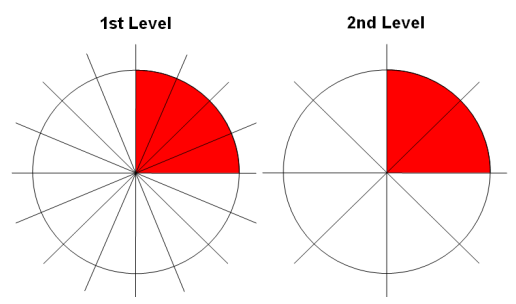

Figure 2: Angular agglomeration in two dimensions.

As one can observe, the agglomeration is performed between angles belonging to the same quadrant of the directional circle; it is this the unique limitation imposed to this scheme in order the derived superangles as well as the merged ones to have the same sign. This constraint results additionally to the definition of a minimum accepted number of azimuthal and polar control angles; 4 and 2 respectively. Similarly to the spatial scheme, the angular agglomeration is assumed to be complete with the computation of the directional weights and the pixelation coefficients for the new angular levels.

As far as the angular agglomeration multigrid enhanced solution of the RTE is concerned, the same strategy to the spatial scheme is followed. At each cycle eqn (5) is solved for the non-multigrid level (denoted with $m n$ ); the values of radiative intensity are restricted angularly weighted to the next coarser resolution (denoted with $M N$ ), while the flux balances are restricted as angularly summed.

For the coarser discretizations an approximated equation is solved, including the angular forcing function $A^{M N}$, defined as:

$$
A^{M N}=R_{p, \text { restricted }}^{M N}-R_{p}^{M N}\left(I_{p, \text { restricted }}^{M N}\right) .
$$

Since the solution to the coarsest directional "sphere" is obtained, the radiative intensity corrections are prolongated to the next finer resolution similarly to the spatial scheme.

\section{Combined spatial/angular agglomeration multigrid scheme}

A combined spatial/angular agglomeration multigrid scheme was finally developed to enhance the existing finite-volume solver with additional acceleration; it considers the nested implementation of the angular multigrid scheme at each level of the spatial one, as illustrated in Figure 3. The procedure begins with the spatial agglomeration at each partition, while at next the coarser angular resolutions are generated. For the iterative solution, the flux computation is performed in the same way, except for the computation of the spatial/angular forcing function $A_{H}^{M N}$, which is described as: 


$$
\begin{aligned}
& A_{H}^{M N}=\left(I_{R}\right)_{m n}^{M N} R_{P, F A S}^{m n}-R_{P}^{M N}\left(\left(I_{I}\right)_{m n}^{M N} I_{P}^{m n}\right)=\left(I_{R}\right)_{m n}^{M N}\left(R_{P}^{m n}+A_{H}^{m n}\right), \\
& -R_{P}^{M N}\left(\left(I_{I}\right)_{m n}^{M N} I_{P}^{m n}\right) .
\end{aligned}
$$

In Figure 3, the bullets denote the positions where the Runge-Kutta (RK) method is implemented in each multigrid cycle; As it can be observed, it is applied both at the beginning and at the end of the angular multigrid cycle; this is necessary in order the values of radiative intensity and flux balances to be updated and consequently smoothed before being prolongated spatially.

\section{Numerical results}

For the evaluation of the proposed algorithm two benchmark test cases were examined and presented in this paper, considering a quasi-3D cubic enclosure, examined initially by Kim and Lee [12], and a 3D tetrahedral enclosure, encountered previously by Murthy and Mathur [13]. The simulation results are compared with the corresponding ones of the reference solvers via the distributions of dimensionless incident radiative heat flux $Q^{*}[5,10]$. For the assessment of the achieved acceleration by the proposed multigrid scheme the radiative intensity residual is computed for each cycle (iteration for a single-grid) at the spatial/angular finest (1st) multigrid level as

$$
\text { residual }=\sum_{P=1}^{N_{P}} \sum_{m=1}^{N_{\theta}} \sum_{n=1}^{N_{\varphi}}\left|I_{P}^{m n, l+1}-I_{P}^{m n, l}\right| / N_{P} N_{\theta} N_{\varphi}
$$

where $N_{P}$ is the number of the nodes of the finest/initial grid. The notation SxAy is used to define the applied multigrid scheme, where $\mathrm{x}$ indicates the utilized spatial multigrid levels while y denotes the angular ones, i.e. S3A2 corresponds to three spatial and 2 angular multigrid levels while S1A1 to a single-grid simulation.

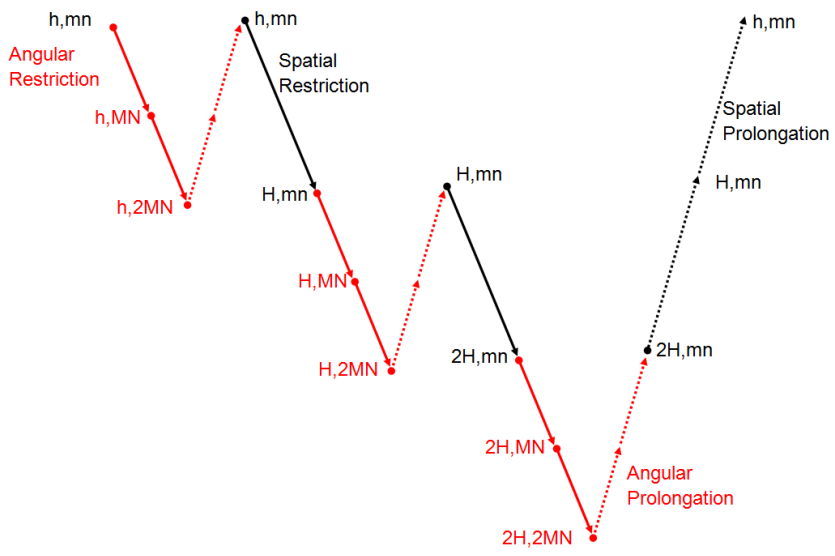

Figure 3: Combined spatial/angular agglomeration multigrid cycle. 


\subsection{A cubic enclosure}

The first quasi-3D test case concerns radiative heat transfer in a cubic enclosure with edge length equal to $1 \mathrm{~m}$ (Figure 4 ), filled with a purely anisotropically scattering medium (extinction coefficient $\beta$ and scattering albedo $\omega$ equal to unity) [12]. The medium as well as the walls are assumed to be cold, except for the bottom face $\left(z_{w}=0 \mathrm{~m}\right)$ at which a constant heating energy is implemented $\left(E=1 \mathrm{~W} / \mathrm{m}^{2}\right)$ and the faces normal to x-direction, where symmetry boundary conditions are imposed. For the angular discretization 16 azimuthal and 8 polar angles were employed, while the overhang problem was alleviated by implementing the pixelation method. The mesh utilized for this simulation is composed of 13,433 nodes and 71,589 tetrahedrons, while for parallelization purposes it was divided in 6 partitions.

For the evaluation of the proposed methodology three sub-cases were encountered, considering different values of wall emissivity $\varepsilon_{w}(1,0.5$ and 0.1 respectively). The Runge-Kutta method was applied along with a second-order accurate spatial scheme, jointed with the Min-mod limiter on a Workstation with an AMD FX ${ }^{(\mathrm{tm})}-8350$ eight-core processor at $4.00 \mathrm{GHz}$. Figure 4 presents the cubic geometry as well as the obtained distributions of incident radiative heat flux $Q^{*}$ along the $\mathrm{A}-\mathrm{A}$ line, compared with the corresponding results of the reference DOM (Discrete Ordinates Method) solver of Kim and Lee [12]. In Figure $5 \mathrm{a}-\mathrm{d}$ the convergence history per iterations and time of the employed non-multigrid and multigrid schemes is illustrated for the first two sub-cases $\left(\varepsilon_{w}=1\right.$ and $\left.\varepsilon_{w}=0.5\right)$; as expected the obtained acceleration increases with the decrease of the wall emissivity, achieving a maximum temporal speed-up coefficient equal to 4.94 .

Figure 5e-h includes the corresponding history for the last sub-case: Figure 5, e-f, results correspond to $\varepsilon_{w}=0.1$, where a maximum temporal speed-up coefficient equal to 8.12 was achieved using the S4A3 scheme. Additionally, Figure $5 \mathrm{~g}-\mathrm{h}$ illustrates (for the same value of $\varepsilon_{w}$ ), the temporal convergence rates for different spatial and angular multigrid schemes, confirming the contribution of the proposed algorithm to considerably augment the computational performance.
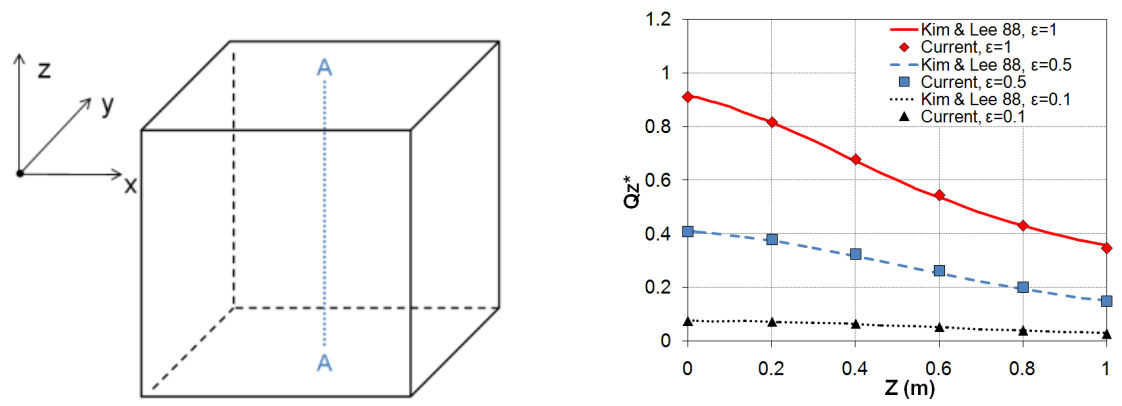

Figure 4: $\quad$ Utilized geometry (left) and distribution of incident dimensionless radiative heat flux $Q^{*}$ along the A-A line (right). 

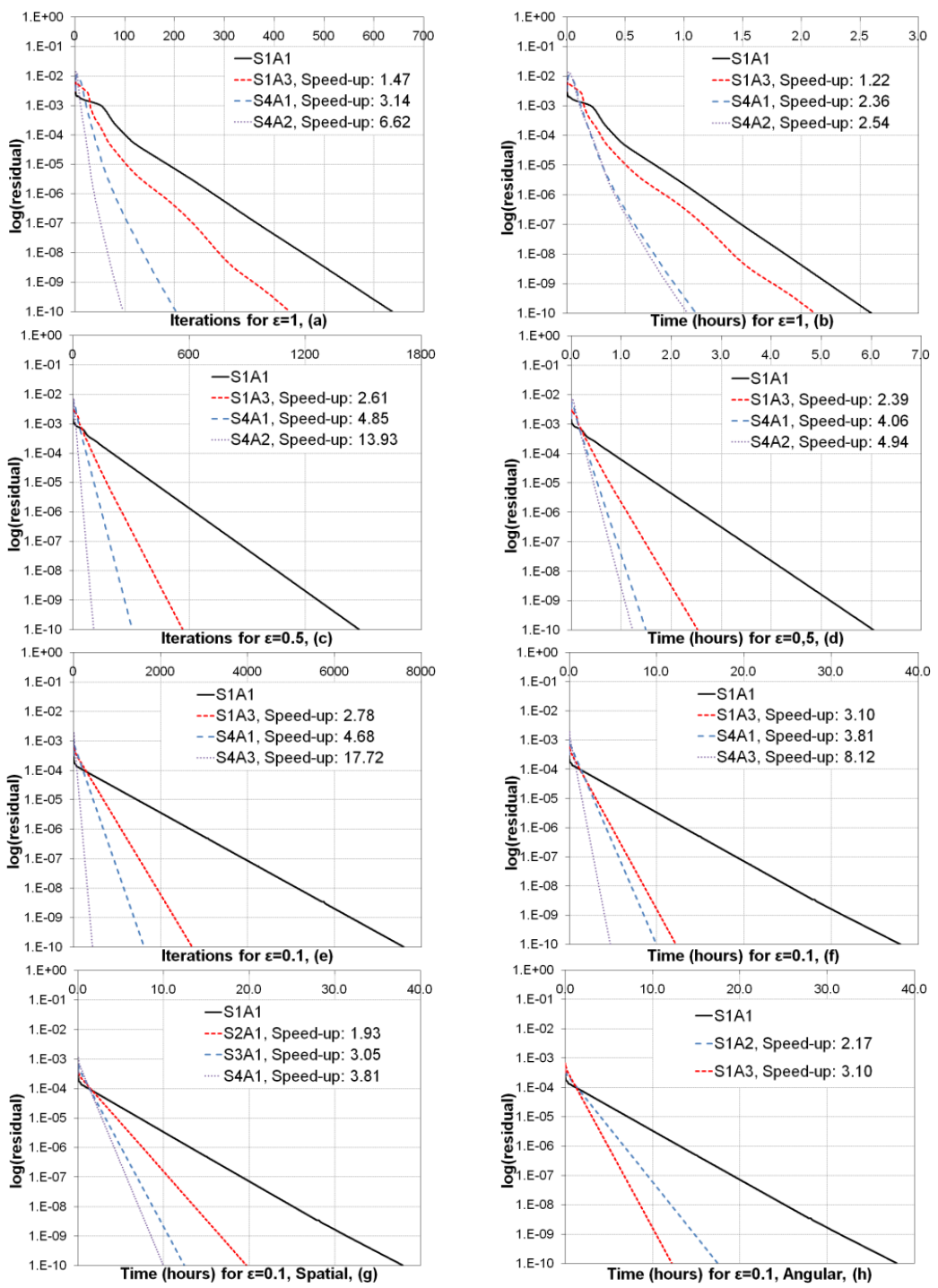

Figure 5: Convergence history for the three sub-cases of the cubic enclosure.

\subsection{A tetrahedral enclosure}

The second test case considers a tetrahedral enclosure with black walls $\left(\varepsilon_{w}=1\right)$, as illustrated in Figure 6 [13]. Although not necessarily required, a relatively fine grid was used, composed of 196,847 nodes and 1,119,456 tetrahedrons, divided in two partitions for parallelization; this mesh was used in order to amplify the effect of the multigrid method and evaluate its performance in large grids. For angular discretization 16 azimuthal and 8 polar angles were employed along with 
the pixelation method. Two different sub-cases were encountered, employing a first-order accurate spatial scheme on a Workstation with an AMD FX ${ }^{(\mathrm{tm})}-8120$ eight-core processor at $3.1 \mathrm{GHz}$. For the first one an absorbing-emitting medium $\left(k_{a}=1 \mathrm{~m}^{-1}, \sigma_{s}=0 \mathrm{~m}^{-1}\right)$ maintained at constant temperature of $100 \mathrm{~K}$ is considered, while all the walls are assumed to be cold; the distribution of the incident dimensionless radiative heat flux $Q^{*}$ along the A-A line is presented in Figure 6, very well compared with the corresponding one of Murthy and Mathur [13]. In Figure $7 \mathrm{a}-\mathrm{b}$ the convergence history per iterations and time is illustrated for all the employed spatial multigrid schemes, obtaining a maximum temporal speedup coefficient equal just to 1.95 , due to the absence of medium's scattering.
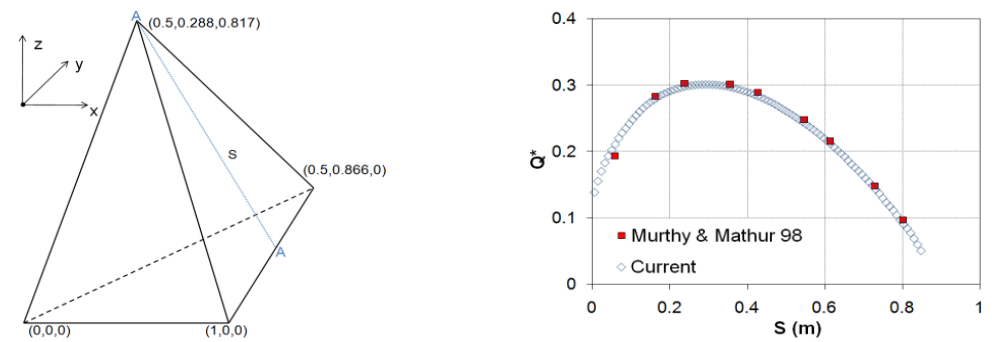

Figure 6: Geometry (left) and distribution of flux $\mathrm{Q}^{*}$ along the A-A line (right).
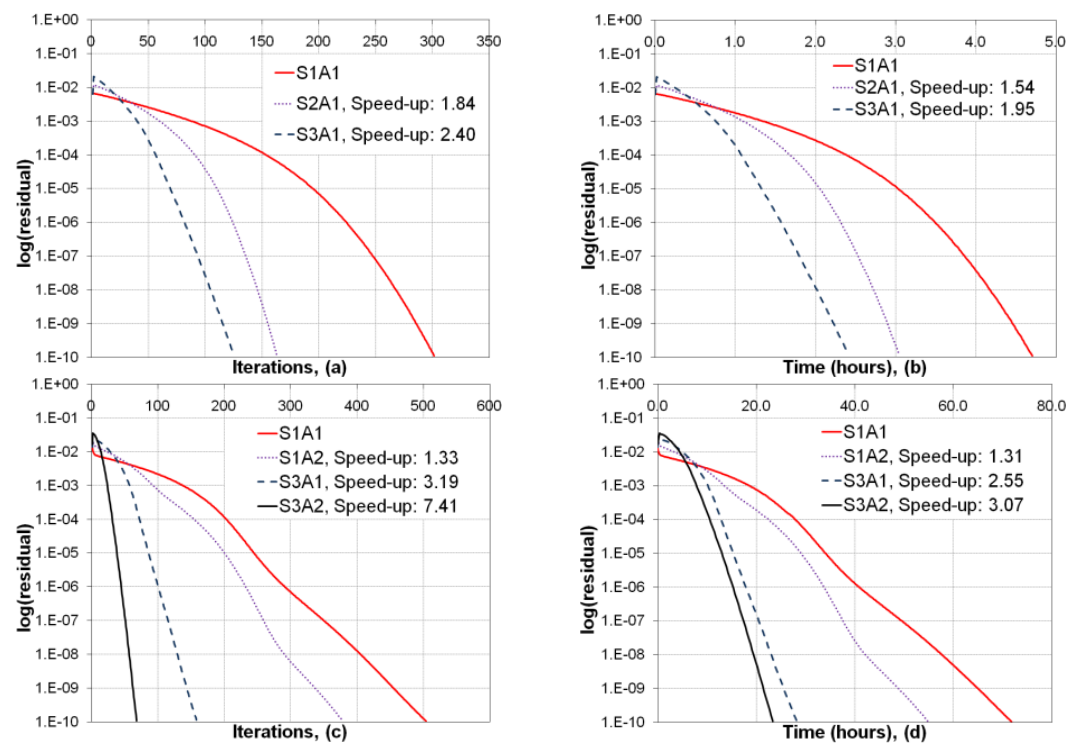

Figure 7: Convergence history for the two sub-cases of the tetrahedral enclosure.

The second sub-case concerns a purely scattering cold medium $\left(k_{a}=0 \mathrm{~m}^{-1}\right.$, $\sigma_{s}=1 \mathrm{~m}^{-1}$ ) and cold walls, except for the face including the A-A line, which is maintained at constant temperature of $100 \mathrm{~K}$. Figure $7 \mathrm{c}-\mathrm{d}$ presents the obtained 
convergence history along with the speed-up rates (maximum in time: 3.07 for S3A2), revealing once more the capability of the algorithm for enhanced acceleration of the solution procedure, when a scattering medium is assumed.

\section{References}

[1] Raithby, G.D. \& Chui, E.H., A finite-volume method for predicting a radiant heat transfer in enclosures with participating media. J. Heat Transfer, 112, pp. 415-423, 1990.

[2] Carre, G. \& Lanteri, S., Parallel linear multigrid by agglomeration for the acceleration of 3D compressible flow calculations on unstructured meshes. Numerical Algorithms, 24, pp. 309-332, 2000.

[3] Mavriplis, D.J. \& Pirzadeh, S., Large-scale parallel unstructured mesh computations for 3D high-lift analysis. NASA ICASE Report, 99-9, 1999.

[4] Blazek J., Computational fluid dynamics: principles and applications (Chapter 9), Elsevier Science, pp. 305-320, 2001.

[5] Lygidakis, G.N. \& Nikolos, I.K., Using a high-order spatial/temporal scheme and grid adaptation with a finite-volume method for radiative heat transfer, Num. Heat Transfer B, 64(2), pp. 89-117, 2013.

[6] Coelho, P.J., Bounded skew high-order resolution schemes for the discrete ordinates method, J. Computational Physics, 175, pp. 412-437, 2002.

[7] Hassanzadeh, P. \& Raithby, G.D., Finite-volume solution of the second order radiative transfer equation: accuracy and solution cost, Num. Heat Transfer B, 53, pp. 374-382, 2008.

[8] Ferziger, J.H. \& Peric, M., Computational methods for fluid dynamics (Chapter 11), 3rd Edition, Springer, pp. 344-351, 2002.

[9] Lallemand, M.H., Schemas decentres multigrilles pour la resolution des equations d'Euler en elements finis, $\mathrm{PhD}$ Thesis, Universite de Provence, France, 1988.

[10] Lygidakis, G.N. \& Nikolos, I.K., Using the finite-volume method and hybrid unstructured meshes to compute radiative heat transfer in 3-D geometries, Num. Heat Transfer B, 62(5), pp. 289-314, 2012.

[11] Nishikawa, H. \& Diskin, B., Development and application of parallel agglomerated multigrid methods for complex geometries, AIAA 2011-3232, Proc. 20th AIAA Computational Fluid Dynamics Conference, Honolulu, Hawaii, USA, 27-30 June, 2011.

[12] Kim, T.K. \& Lee, H., Effect of anisotropic scattering on radiative heat transfer in two-dimensional rectangular enclosures, Int. J. Heat Mass Transfer, 31, pp. 1711-1721, 1988.

[13] Murthy, J.Y. \& Mathur, S.R., A finite volume method for radiative heat transfer using unstructured meshes, AIAA 98-0860, Proc. 36th Aerospace Sciences Meeting and Exhibit, Reno, USA, 12-15 January, 1998. 